
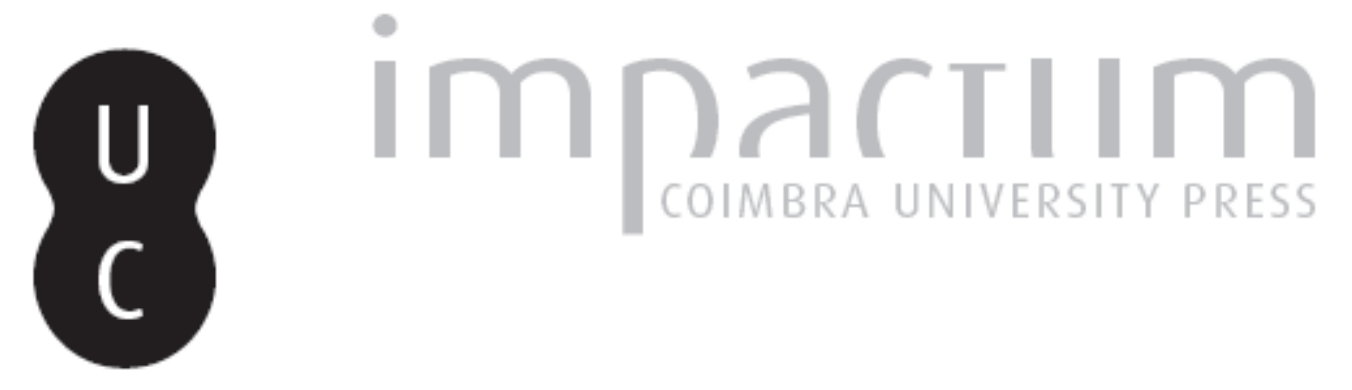

\title{
Traumatismo ocular grave por airbag
}
Autor(es):
Lorenzo AE, Hernando; Anduaga A, Menchaca; GómezGuillamón F, GarcíaNieto; Anaya P, Moreno

Publicado por: Imprensa da Universidade de Coimbra

URL persistente:

URI:http://hdl.handle.net/10316.2/33223

DOI:

DOI:http://dx.doi.org/10.14195/1647-8630_24_4

Accessed : $\quad$ 26-Apr-2023 16:33:06

A navegação consulta e descarregamento dos títulos inseridos nas Bibliotecas Digitais UC Digitalis, UC Pombalina e UC Impactum, pressupõem a aceitação plena e sem reservas dos Termos e Condições de Uso destas Bibliotecas Digitais, disponíveis em https://digitalis.uc.pt/pt-pt/termos.

Conforme exposto nos referidos Termos e Condições de Uso, o descarregamento de títulos de acesso restrito requer uma licença válida de autorização devendo o utilizador aceder ao(s) documento(s) a partir de um endereço de IP da instituição detentora da supramencionada licença.

Ao utilizador é apenas permitido o descarregamento para uso pessoal, pelo que o emprego do(s) título(s) descarregado(s) para outro fim, designadamente comercial, carece de autorização do respetivo autor ou editor da obra.

Na medida em que todas as obras da UC Digitalis se encontram protegidas pelo Código do Direito de Autor e Direitos Conexos e demais legislação aplicável, toda a cópia, parcial ou total, deste documento, nos casos em que é legalmente admitida, deverá conter ou fazer-se acompanhar por este aviso.

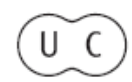


RE VISTA P O R T U G U E S A
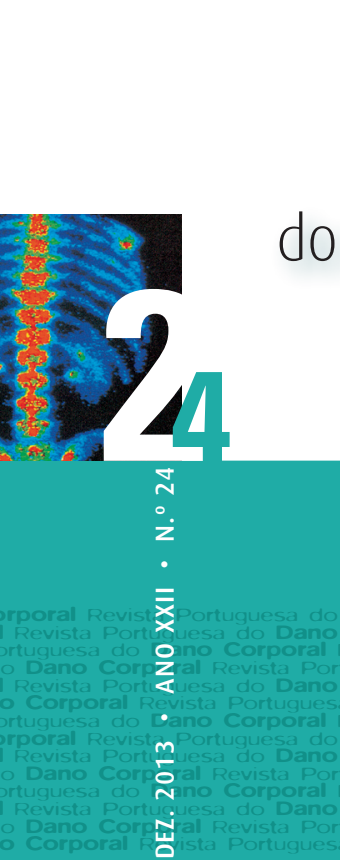

$$
\text { RE VISTA P OR TU/G U E S A }
$$

do

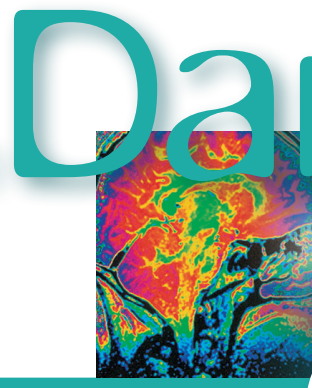

ন

$\stackrel{\circ}{\dot{z}}$

$\dot{\bar{x}}$

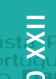

Ono Corporal Revistaral Revista Portuming

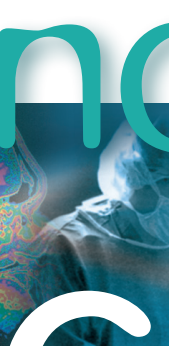

(9)

12

tै. strentes

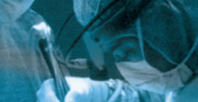

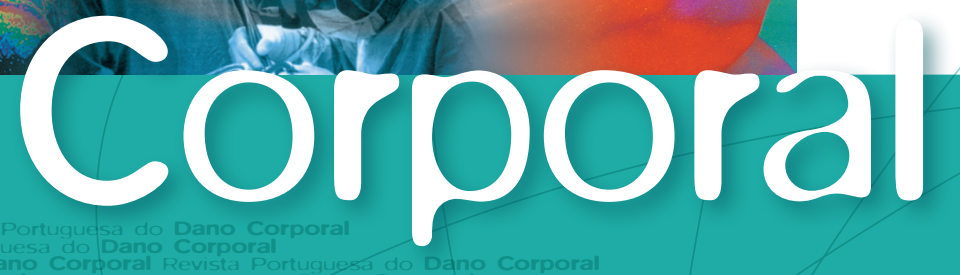

-

m Portuguesa do Dano Corporal Revista Portuguesa do Qano Corporal

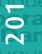
ristal

Corporal Revista Portuquesa do Dano Corpograi Revista Portuquesa do Dang Corporal

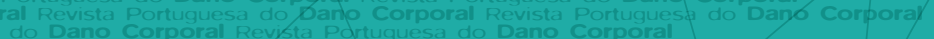

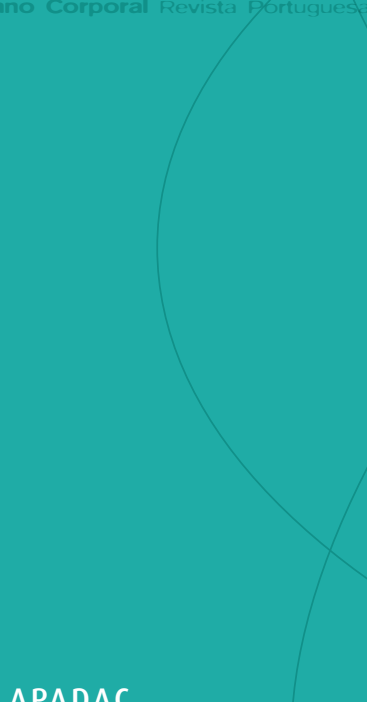

APADAC

ASSOCIAÇÃO PORTUGUESA

DE AVALIAÇÃO

DO DANO CORPORAL

FACULDADE DE MEDICINA

DA UNIVERSIDADE

DE COIMBRA 


\title{
Traumatismo ocular grave por airbag
}

\author{
Hernando Lorenzo AE ${ }^{1}$, Menchaca Anduaga $A^{2}$, García-Nieto Gómez-Guillamón F1, \\ Moreno Anaya $\mathrm{P}^{3}$
}

\section{DESCRIPCION DEL CASO}

Se trata de una mujer de 44 años, ocupante del asiento delantero derecho de un turismo BMW modelo 320, que sufre accidente de tráfico por la noche, (salida de vía con choque frontal derecho contra barrera bionda de protección de la carretera). El automóvil presentaba daños en su parte frontal derecha. (Fotografía No. 1)

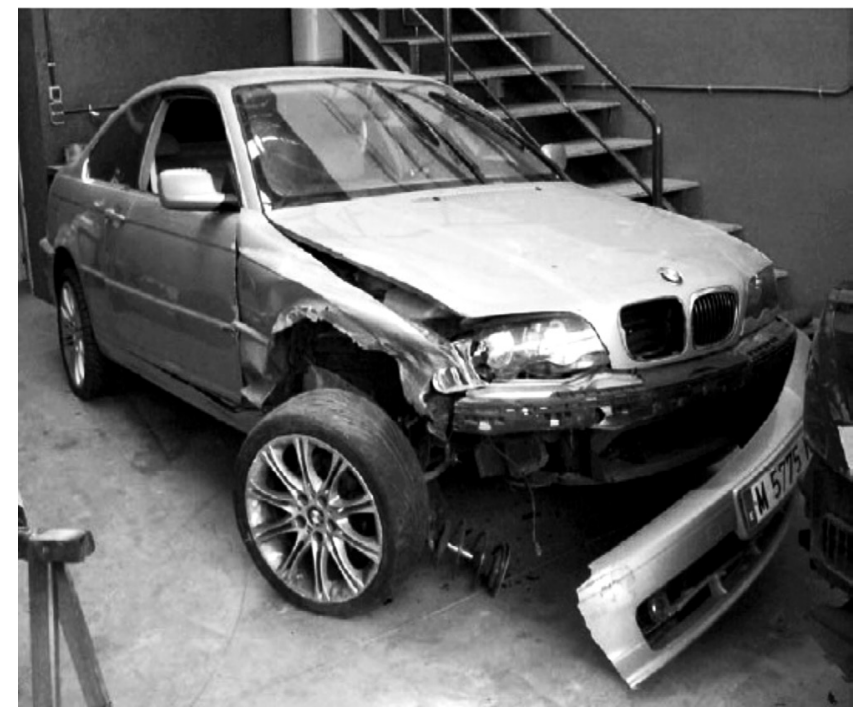

Foto No. 1 - Daños en zona frontal-lateral derecha del turismo siniestrado

Servicio de Medicina Intensiva, H. U. 12 de Octubre, Madrid

Dirección de Enfermería, SUMMA 112, Madrid

Servicio de Radiodiagnóstico, H. U. "La Paz", Madrid 
La conductora del turismo (mujer de 22 años), quien también utilizaba cinturón de seguridad, no sufrió lesiones.

La lesionada viajaba con cinturón de seguridad y se activaron los dos airbags delanteros (de conductora y de acompañante) y el airbag lateral derecho; esta ocupante viajaba con su asiento en posición adelantada. Tras el accidente sufrió traumatismo facial con estallido ocular derecho, fractura amplia de suelo orbitario derecho, heridas en la cara y laceración de pabellón auricular derecho, (helix).

Tanto en el atestado policial, como en la inspección realizada por los peritos reconstructores (ingeniero y médico), se acreditó que se habían activado los airbags delanteros del turismo (de conductor y de acompañante), y el airbag lateral delantero derecho (de acompañante), fotografía No. 2.

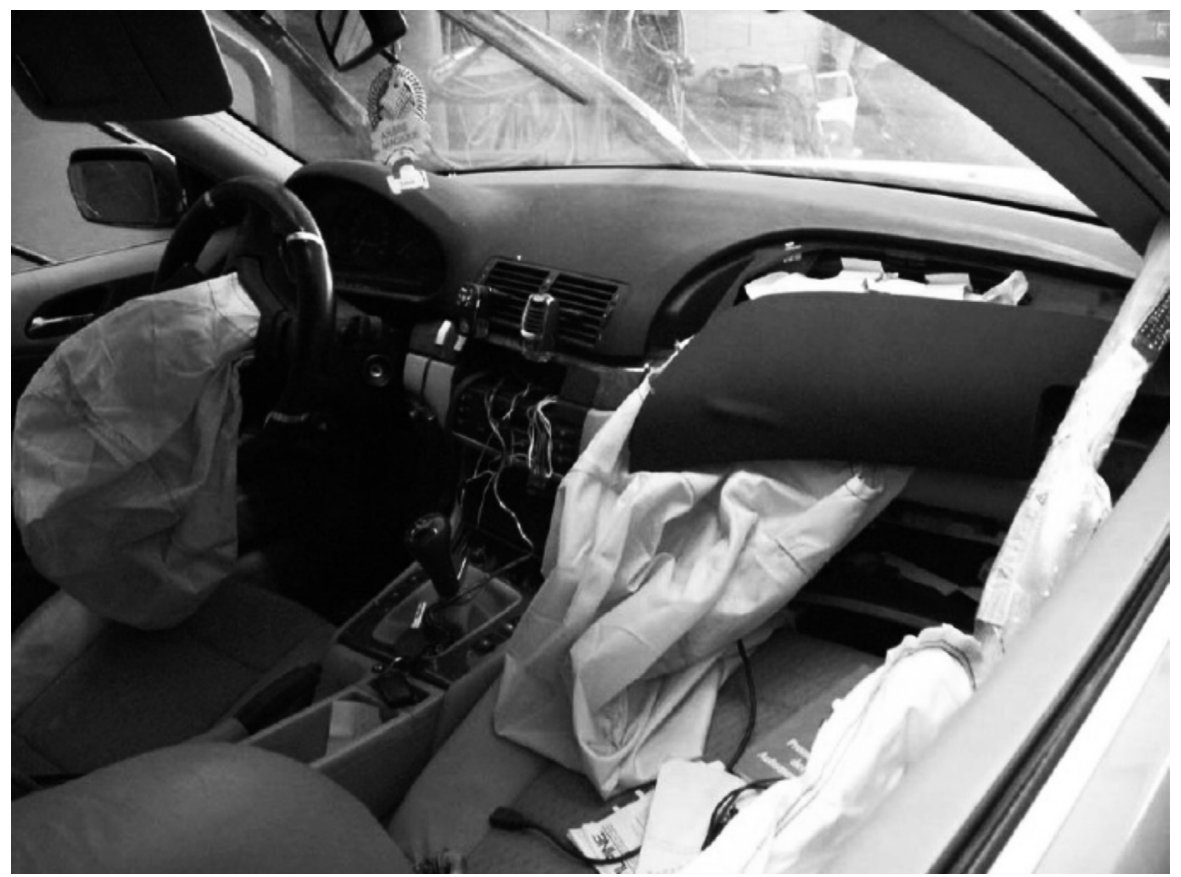

Foto No. 2 - Airbags delanteros y lateral derecho activados

Estas lesiones son compatibles con la activación de los airbags de la acompañante, siendo las lesiones oculares y la fractura de órbita derecha, a nivel de suelo, favorecidas por la circunstancia de que esta ocupante habría viajado muy próxima a la guantera donde se alojaba el airbag delantero, que al activarse habría provocado una "explosión" produciendo las lesiones faciales y oculares descritas. (Existía una pequeña mancha de sangre en el airbag frontal de la acompañante), fotografia No. 3. 


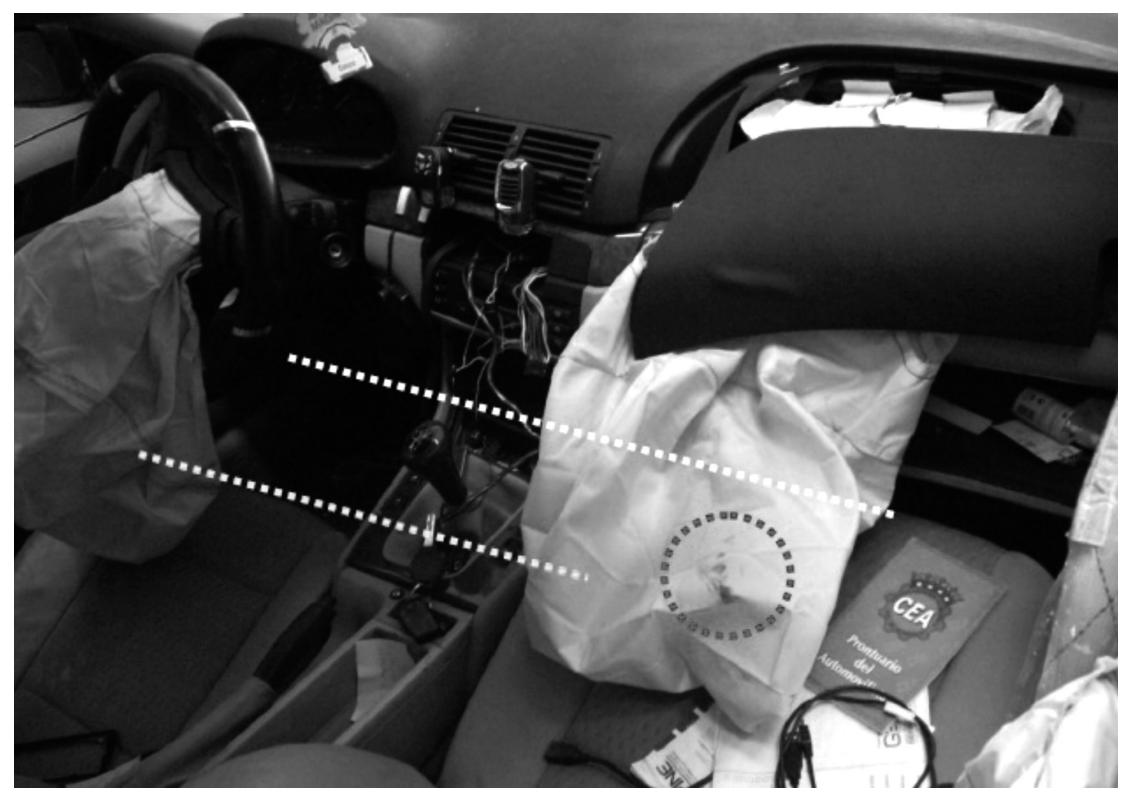

Foto No. 3 - Mancha de sangre en airbag delantero derecho y posición adelantada del asiento

\section{DISCUSION Y COMENTARIOS}

Crandall CS y cols. ${ }^{1}$ valoraron la eficacia de los sistemas de protección de ocupantes, para lo que midieron la reducción de mortalidad asociada al despliegue de airbag y al uso de cinturón de seguridad en conductores que habían sufrido accidentes de automóvil con colisión frontal entre vehículos, en los EE.UU. Hubo 9.859 colisiones frontales que afectaron a 19.718 ocupantes y conductores de automóviles.

Utilizaron un diseño caso-control de todas las colisiones frontales que afectaban a dos automóviles, referidas por el FARS -Sistema de Referencia de Análisis de Mortalidad- en el periodo 1992-1997 y las diferencias de mortalidad en conductores entre los vehículos accidentados mediante comparación por parejas para despliegue de airbag y utilización de cinturón de seguridad y estableciendo la relación correspondiente.

El despliegue de airbag redujo la mortalidad en un $63 \%$, mientras que el uso de cinturón de seguridad de fijación en tres puntos -con banda torácica y abdominal- redujo la mortalidad en un $72 \%$. El uso combinado de airbag y cinturón de seguridad redujo la mortalidad en más del 80\%. Por lo tanto este estudio confirma el efecto independiente de los airbag y los cinturones de seguridad en la reducción de la mortalidad. 
Los hallazgos demuestran una reducción dramática en el riesgo de muerte por colisiones frontales en conductores que utilizan sus cinturones de seguridad y en los que se despliega el airbag.

Evans $\mathrm{L}^{2}$ respecto a la efectividad de los cinturones de seguridad para evitar muertes en accidentes de tráfico, utilizó la metodología del doble par, para calcular la eficacia de los cinturones de seguridad. Comparando la mortalidad en conductores con la de otros ocupantes en el mismo vehículo, fue capaz de calcular una diferencia de mortalidad prácticamente a la mitad, atribuible al uso de cinturón de seguridad.

Estudios previos al de Crandall y cols. han calculado la eficacia de los airbag comparando las tasas de mortalidad en accidentes frontales y no frontales ${ }^{3}$.

Otros estudios que han medido la reducción de mortalidad debido a airbag, han calculado una reducción del $24 \%$ al $28 \%$ en colisiones frontales ${ }^{4}$. Esta utilidad ha sido confirmada por otros autores ${ }^{5,6}$.

Junto con el cinturón de seguridad, el airbag es un elemento de seguridad pasiva indispensable en los automóviles modernos. Se estima que en caso de impacto frontal de un vehículo su uso puede reducir el riesgo de muerte en un 30\%. Por otra parte, debe recordarse que el airbag es un elemento de seguridad pasiva complementario al cinturón de seguridad. Así, las siglas SRS que figuran en los airbags, significan $\mathbf{S}=$ Supplemental -Complementario-, $\mathrm{R}$ = Restraint, -Sujeción- y $\mathbf{S}$ = System, -Sistema-, es decir, Sistema de Sujeción Complementario (al cinturón de seguridad).

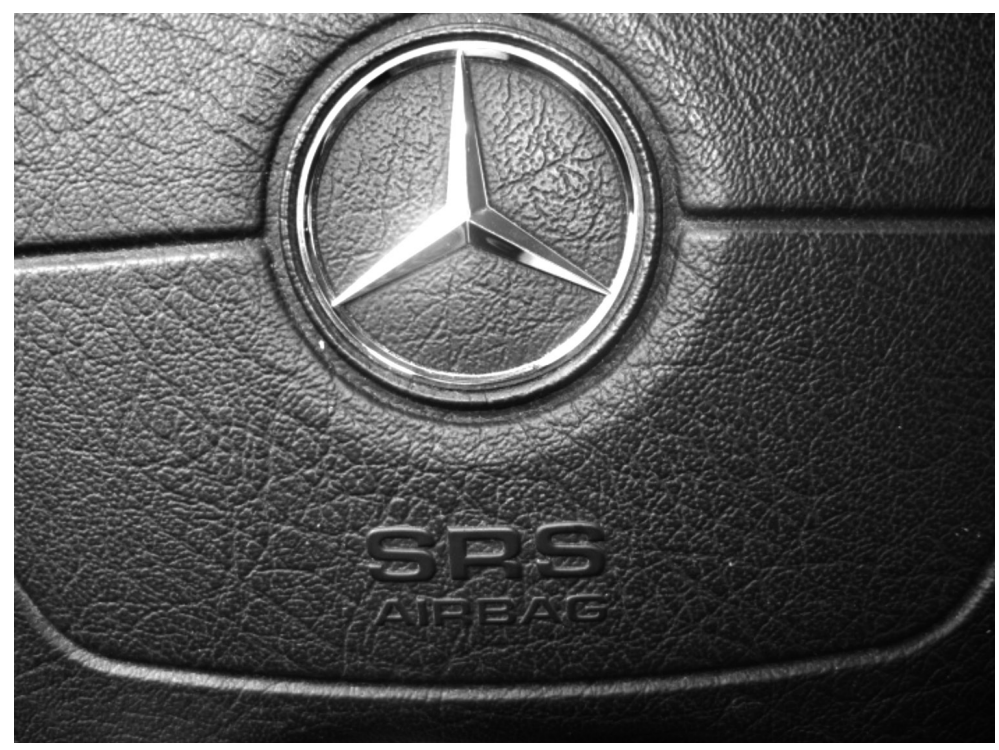


Para detener un objeto que está en movimiento, es necesaria la acción de una fuerza actuando durante cierto tiempo en sentido opuesto a ese movimiento. Cuanto más rápida sea la parada, más intensa tiene que ser la fuerza. Si, por el contrario, la parada se produce en un periodo de tiempo prolongado, la fuerza de retención puede ser menor.

El objetivo del airbag es detener el cuerpo de los ocupantes de un vehículo lo más suavemente posible. Esto no es fácil, pues el sistema sólo dispone del espacio existente entre el conductor y el volante; y de un tiempo de centésimas de segundo. A pesar de todo, prolongar o amortiguar, "dosificar" la parada de los ocupantes en un tiempo y un espacio tan reducidos crea sobre sus cuerpos fuerzas menores de las que sufrirían si la parada fuera instantánea. Es decir, el airbag permite amortiguar el golpe del cuerpo contra el volante, el salpicadero y el parabrisas.

Para cumplir ese cometido, el airbag hace uso de los siguientes elementos:

- Una bolsa (bag) o cojín inflable, fabricado en nailon, el cual está plegado en el centro del volante, en el salpicadero o en cualquier otro lugar donde sea necesario introducir un efecto amortiguador del golpe.

- Un detector de impacto que determina cuándo se produce un choque y activa el inflado del airbag.

- Un sistema de inflado, basado en una reacción química que se produce de modo casi explosivo y da lugar a un gran volumen de gas nitrógeno. Esta reacción es activada por sistema eléctrico controlado por el detector de impacto.

Los gases producidos de modo explosivo alcanzan suficiente presión como para inflar el airbag en 20 centésimas de segundo. La rapidez del proceso es tal, que el volumen de gas producido hace que el airbag salga de su alojamiento a una velocidad de $300 \mathrm{~km} / \mathrm{h}$. Instantes después de que el airbag se infle, el gas producido comienza a disiparse a través de pequeños orificios existentes en la tela. De este modo, el airbag se desinfla permitiendo la movilidad de los ocupantes.

Aunque se ha demostrado un efecto beneficioso del airbag para reducir lesiones en ocupantes de automóviles, tras sufrir accidentes, también se han descrito lesiones atribuidas a este dispositivo de seguridad pasiva ${ }^{7}$.

Las lesiones oculares durante accidentes de tráfico supusieron el $8 \%$ de todas las lesiones oculares referidas en el registro de Ojos de los EE.UU entre 1982 y $1989^{\circ}$. Solamente el $7 \%$ de estas lesiones oculares se relacionaban con airbags ${ }^{9}$. 

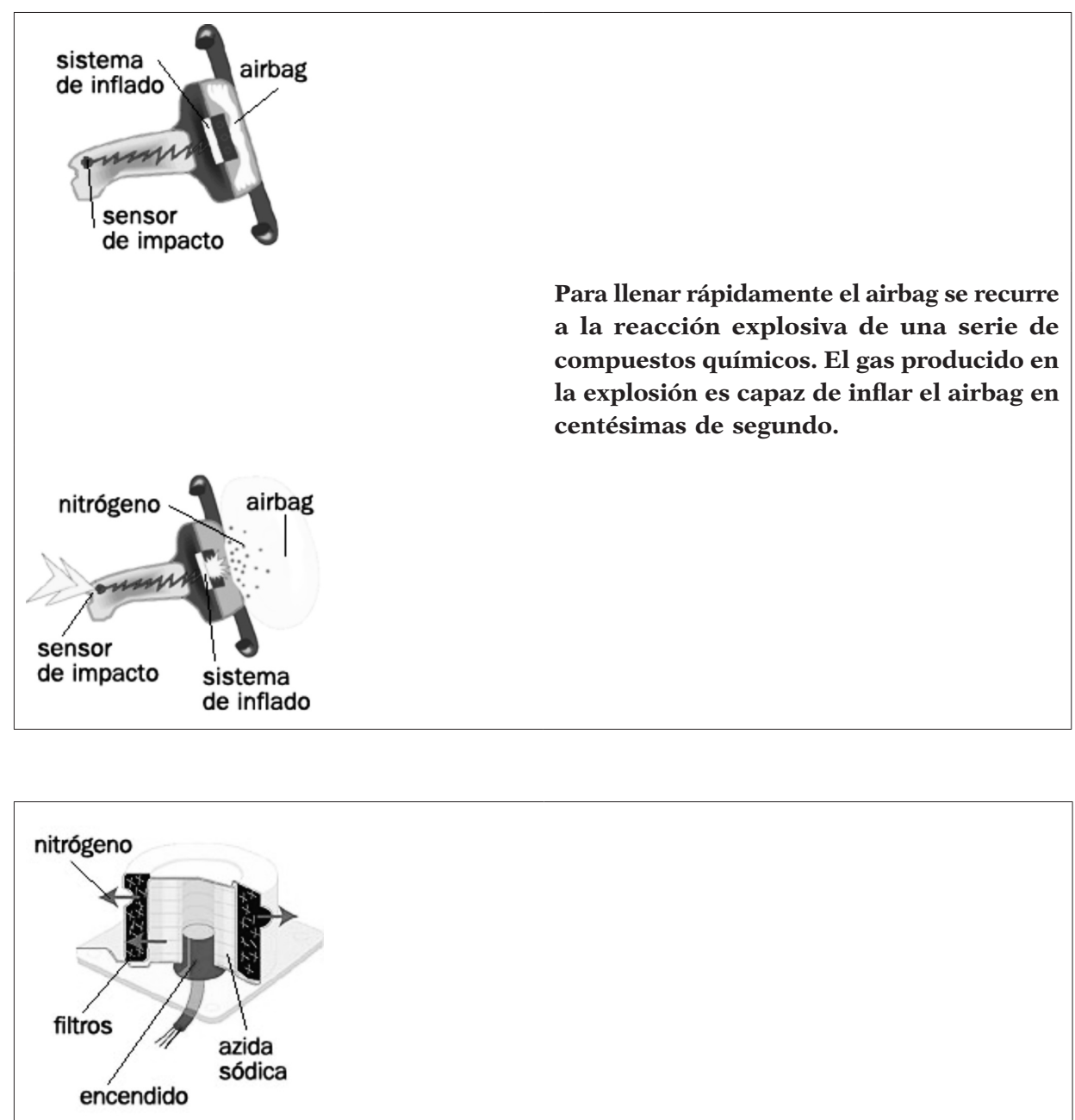

Dispositivo del sistema de inflado de un airbag

Respecto a las lesiones oculares por airbag, Duma SM y Crandall JR ${ }^{10}$, refieren que los diseños de airbag tradicionalmente hacían el despliegue del airbag a través de una puerta con una junta para separarlo del salpicadero. La tendencia reciente en diseño hace que se haya retirado la puerta y el airbag se coloca detrás de un panel, sin tapadera.

Se ha visto que con la introducción de las cubiertas del módulo de airbag sin tapadera, se presenta un riesgo adicional de lesión ocular por las partículas de esponja, que no precisa un contacto directo con el airbag.

Históricamente, las lesiones oculares por airbag se han atribuido al contacto del ojo con el airbag desplegándose o ya desplegado. También 
se han referido casos de lesiones indirectas producidas por interacción del airbag con gafas o por ejemplo con una pipa de fumador, siendo estas lesiones muy raras.

Se han descrito lesiones oculares bilaterales por airbag, planteándose que el uso de gafas podría constituir un riesgo adicional ${ }^{11}$.

Vichnin MC, Jaeger EA, Gault JA et al ${ }^{12}$ presentaron resúmenes de 14 casos en los cuales las lesiones oculares más frecuentes debidas a airbag eran los hifemas, que se definen como el almacenamiento de sangre en la cámara anterior, y las erosiones corneales.

Manche EE, Goldgerg $\mathrm{RA}^{13}$ revisaron cinco casos, y encontraros hifemas en todos los casos así como contusiones, roturas de globo y desprendimientos de retina.

El traumatismo de párpado, las erosiones corneales y las hifemas fueron las lesiones más prevalentes en los 11 casos estudiados por Ghafouri $\mathrm{A}^{14}$. Aunque la mayoría de casos se debían a despliegue del airbag lateral de conductor, documentaba lesiones oculares como resultado del despliegue de un airbag lateral de pasajero.

Larkin $\mathrm{GL}^{15}$ refirió la primera lesión corneal producida por airbag.

En las pruebas de choque estándar (un automóvil que golpee contra un muro a una velocidad de $50 \mathrm{Km} / \mathrm{h}$ ) el inflado del airbag comienza a los 15 milisegundos después del impacto. El gas de nitrógeno hincha el airbag, rompiendo la cubierta y la bolsa se expande hacia delante. 50 milisegundos después del impacto el airbag finaliza su expansión y mantiene brevemente un volumen y una presión constantes expulsando el gas por dos agujeros de salida situados en la parte trasera de la bolsa.

Un pasajero que se sienta en posición normal y utilice cinturón de seguridad no impactará contra el airbag al menos hasta después de 60 milisegundos después de una colisión y esto le protegerá de golpear directamente contra el volante o el parabrisas. Por otra parte, una posición del conductor muy próxima al cartucho del airbag, podría impactar el airbag durante el proceso de inflado y expansión. El autor colocó al maniquí a distancias que variaban de 160 a 320 mms de distancia del cartucho del airbag y observaron pérdida de células endoteliales ligeramente mayor a la distancia de $240 \mathrm{mms}$, que fue la distancia que utilizaron para todas las pruebas siguientes. Este efecto puede estar producido por el despliegue de la parte anterior del airbag que se refiere que alcanza una velocidad máxima a aproximadamente los $200 \mathrm{mms}$ del cartucho.

El airbag se dispara cuando existe un impacto frontal con una angulación de $30^{\circ}$ o menos respecto a la línea que recorre longitudinalmente al automóvil por su parte central de delante a atrás, y cuando un sensor electrónico de colisión detecta una desaceleración repentina desde una velocidad de aproximadamente $32 \mathrm{Km} / \mathrm{h}$. 
Si las condiciones de colisión del sensor se cumplen, se dispara un inflador, que es un dispositivo pirotécnico que contiene nitrato sódico, que bajo ignición produce un gran volumen de gas nitrógeno, el cual entonces infla la bolsa de aire hasta un volumen de 40 litros (en el diseño europeo o Eurobag) o 70 litros (airbag de tamaño completo) en aproximadamente 50 milisegundos. La velocidad máxima de la bolsa durante el despliegue está entre unos 75 y unos $260 \mathrm{Km} / \mathrm{h}$ pero debe ser cero en el momento de contacto con un conductor que esté colocado normalmente.

O'Hullomun HS $^{16}$ refiere el caso de un ocupante de asiento delantero derecho, de 76 años, que sufrió un accidente de tráfico leve. No presentaba fracturas orbitarias y tras haberse activado el airbag, había múltiples erosiones cutáneas perioculares y un desgarro profundo que se extendía $3 \mathrm{cms}$ por debajo de la ceja izquierda.

Las lesiones encontradas, (desgarro extenso corneo-esclerótico con un globo hipotónico, y en la operación globo aplanado con una presión intraocular no registrable, cristalino extruido y opaco y localizado en el formix inferior, desgarro corneo-escleral de espesor completo, prolapso extenso de tejido uveal a través de la herida y cámara anterior y cavidad vítrea deformadas), obligaron a una enucleación primaria con inserción de un implante de metacrilato polimetilo.

Scott IU, John GR y Stark $\mathrm{WJ}^{17}$ referían que hasta esa fecha se habían referido tres lesiones oculares asociadas a airbag con lesiones directamente atribuibles al airbag. Las lesiones incluían abrasiones faciales y corneales, hifemas y hemorragias intraoculares.

Los autores describen una paciente que sufrió lesiones oculares graves, con fracturas periorbitarias, desgarros de retina y subluxación de cristalino directamente relacionadas a un airbag hinchado.

Se refiere el caso de una conductora de 64 años quien a una velocidad de unos $70 \mathrm{Km} / \mathrm{h}$ golpeó el quitamiedos en una carretera. Se hinchó el airbag del conductor durante el accidente.

El daño del automóvil se limitaba al parachoques y a la zona guardaparachoques de la derecha. La paciente sufrió abrasiones faciales en el lado derecho y un desgarro en el párpado inferior derecho. La exploración mostró erosiones periorbitarias en el lado derecho y equimosis con movimientos extraoculares intactos y otra exploración con la lámpara de hendidura demostró quemosis y un hifema del 5\%. La TAC mostró fracturas en los huesos nasales derecho e izquierdo, con opacificación de celdillas etmoidales y un nivel hidroaéreo en el seno maxilar derecho que evidenciaban fracturas de las paredes medial o interna y del suelo de la órbita derecha. Exploraciones posteriores mostraron subluxación superior del cristalino asociada a desplazamiento anterior del vítreo.

Duma $\mathrm{SM}^{18}$ refiere que las lesiones oculares inducidas por airbag pueden dividirse en dos tipos. El primero se origina por aspectos mecánicos del airbag 
cuando se despliega en incluye lesiones tales como abrasiones, hemorragias de vítreo, hifemas y desgarros retinianos y desprendimiento de retina. La pérdida de células endoteliales corneales como resultado de la deformación del globo ocular durante el impacto también se incluye en esta categoría.

El segundo tipo es la queratitis química alcalina producida por la deposición sobre el ojo de hidróxido sódico y esto se produce por la salida del nitrato sódico que se está quemando utilizado para producir nitrógeno para el hinchado del airbag.

De los 25 casos descritos, 22 correspondían a conductores con cinturón de seguridad, 1 sin cinturón, 1 a un ocupante con cinturón y el otro a un ocupante sin cinturón.

El impacto con un airbag produce traumatismo ocular que típicamente produce cuatro tipos generales de lesiones: contusiones, erosiones, desgarros y desprendimientos y roturas relacionadas. Los desprendimientos de retina y las roturas relacionadas se consideran el tipo más grave; se cree que el impacto del airbag crea una onda de choque a través del globo y las estructuras orbitarias. Esta onda de choque se refleja en la parte posterior de la órbita y produce una onda de rarefacción que desgarra los vasos sanguíneos y los nervios cuando pasa de vuelta hacia la parte anterior del ojo.

Las contusiones son un resultado frecuente del impacto por el airbag. En esa serie había 10 casos que mostraban contusiones de la cara, párpado o globo ocular. De ellos sólo uno fue resultado del contacto con el airbag cuando todavía se estaba desplegando. Se define un airbag desplegado como uno que está completamente hinchado antes del contacto con el ocupante, mientras que un airbag que se está desplegando está todavía inflándose en el momento del contacto.

Se han descrito numerosos informes que detallan la producción de lesiones oculares graves producidas por airbag incluso en presencia de utilización de cinturón de seguridad con fijación en tres puntos y en la presente serie, 23 de los 25 casos descritos mostraban lesiones oculares en ocupantes con un cinturón de seguridad abrochado.

A

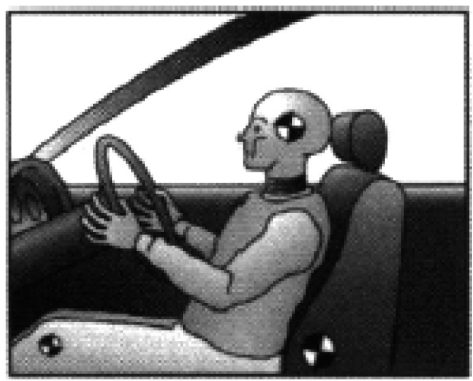

B

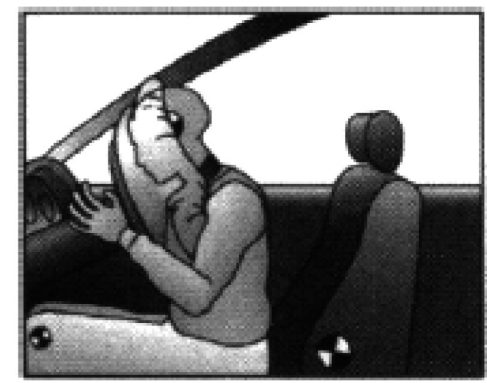


Incluso aunque los automóviles estén dotados de airbag, se debe utilizar cinturón de seguridad. Evans demostró que en los automóviles con airbag, los conductores que utilizan cinturón de seguridad con fijación en tres puntos reducen su riesgo de lesión mortal en un $41 \%$ respecto a los que no lo utilizan ${ }^{19}$.

Stein JD, Jaeger EA, Jeffers $\mathrm{JB}^{20}$ en un artículo clásico revisan la literatura desde 1991 a 1998 e identificaron 44 artículos describiendo 97 pacientes con lesiones oculares producidas por airbag. Hubo abrasiones corneales en el 49\% de los ocupantes lesionados, hifemas en 43\%, hemorragias de vitreo o retinianas en $25 \%$, y desgarros o desprendimientos de retina en $15 \%$. Hubo rotura de globo en 10 pacientes. Los pacientes que habían sufrido accidentes a velocidades mas altas (por encima de $48 \mathrm{kms} / \mathrm{h}$ ), sufrieron un porcentaje más alto de hemorragias de vítreo o retinianas y de cataratas traumáticas, mientras que a velocidades inferiores hubo mayor propensión a desgarros o desprendimientos de retina.

Se han descrito diversos casos de mujeres con un desprendimiento de retina producido por airbag, con buen resultado tras tratamiento ${ }^{21,22,23}$.

Chang-Sue ${ }^{24}$ describe en Taiwan tres pacientes con lesiones oculares por airbag; ninguno de ellos utilizaba cinturón de seguridad, y los tres sufrieron, entre otras lesiones, hemorragia vítrea. Uno de ellos sufrió además desprendimiento de retina.

Aunque se ha demostrado el efecto protector de los airbags en lo referente a reducción de lesiones en ocupantes de automóviles tras colisiones, y debido a las lesiones que se han observado tras su activación, se han realizado esfuerzos para tratar de conseguir el máximo efecto protector a expensas de las mínimas lesiones ${ }^{25}$.

La posición de los ocupantes muy próxima a la zona de ubicación de los airbags, facilitaría la producción de lesiones, tanto en adultos como en niños, que podrían afectar a diferentes zonas corporales - cabeza, cuello, etc ${ }^{26}$.

Los airbags laterales, protegen de lesiones en tórax y abdomen en colisiones laterales y deben inflarse muy rápidamente debido a la escasa distancia entre la parte interior de la puerta y el tronco del ocupante, investigándose el desarrollo de nuevos airbags ${ }^{27}$.

Respecto a las fracturas de órbita, y aunque se han descrito en ocupantes de automóviles tras activación de airbag, este dispositivo reduciría la incidencia de este tipo de lesión, siendo además menos graves las fracturas. Duma SM, Jernigan M. Virginia $\mathrm{MS}^{28}$ refieren en un análisis de 12.429.580 ocupantes de asientos delanteros en 25.464 accidentes tipo colisiones o choques frontales. De todos los ocupantes expuestos a despliegue del airbag, 0,09\% sufrieron fractura de órbita. En contraste con ello, los ocupantes que no se vieron expuestos a airbag tuvieron mas del doble de fracturas orbitarias $(0.22 \%)$. Además de reducir la incidencia, los airbags mostraron un descenso en la 
gravedad de fracturas orbitarias. Estas fracturas de órbita tras activación del airbag, parecen estar relacionadas con una posición adelantada del ocupante lesionado en el momento de la activación del airbag ${ }^{29}$.

Jin-Man $\mathrm{Kim}^{30}$ refiere en Corea un caso muy similar al que nosotros describimos: cita las lesiones producidas en una mujer de 43 años, que utilizaba cinturón de seguridad y cuyo coche chocó contra una barrera, a una velocidad de unos $45 \mathrm{kms} / \mathrm{h}$, produciendo daños en el parachoques del automóvil. La conductora sufrió una fractura periorbitaria, hifema, hemorragia vítrea y rotura coroidea. Onwuzuruigbo ${ }^{31}$ describe una luxación bilateral de cristalino tras activación de airbag, con producción de ceguera bilateral, hemorragias en cámara anterior y erosiones faciales.

\section{Bibliografía}

${ }^{1}$ Crandall CS y cols. Mortality reduction with air bag seat belt use in head-on passenger car collisions. A. J. Epidem, 2001; 153: 219-224.

${ }^{2}$ Evans L. Double pair comparison: a new method to determine how occupant characteristics affect fatality risk in traffic crashes. Accid. Anal. Prev.,1986;18: 217-227.

${ }^{3}$ Zador PL, Circone MA. Automobile driver mortalities in frontal impacts: airbag compared with manual belts. A. J. Public Health, 1993; 83: 661-666.

${ }^{4}$ Lund AK, Ferguson SA. Driver fatalities in 1985-1993 cars with airbags. J. Trauma 1995; 38: 469-475.

${ }^{5}$ Segui-Gomez M. Driver airbag effectiveness by severity of the crash. Am. J. Public Health 2000; 90:1575-1581.

${ }^{6}$ Graham J, Thompson K, Goldie S, Segui-Gomez M, Weinstein M. The cost-effectiveness of airbags by seating position. JAMA - J Am Med Assoc 1997; 278:1418-1425. Reply JAMA-J Am Med Assoc 1998; 279: 506-507.

${ }^{7}$ Wallis LA, Greaves I. Injuries associated with airbag deployment. Emerg. Med. J. 2002; 19:490-493.

${ }^{8}$ Kuhn F, Collins P, Morris R. Epidemiology of motor vehicle crash-related serious eye injuries. Accid. Anal. Prev. 1994; 26:385-390.

9 Joshua D, Edward A, John B. Air bags and ocular injuries. TAOS Annual Meeting. 1999; 97:59-86.

${ }^{10}$ Duma SM, Crandall JR. Eye injuries from airbags with seamless module covers. J. Trauma, 2000; 48: 786-789.

${ }^{11}$ Lee WB, O`Halloran HS y cols. Airbags and bilateral eye injury: five case reports and a review of the literature. J. Emerg. Med., 2001; 20: 129-134.

${ }^{12}$ Vichnin MC, Jaeger EA, Gault JA et al. Ocular injuries related to air bag inflation. Ophthalmic Surg. Lasers 1995: 26: 542-548.

${ }^{13}$ Manche EE, Goldgerg RA. Airbag related ocular injuries. Ophthalmic Surg. 1997; 28: 246-250.

${ }^{14}$ Ghafouri A, Burgess SK, Hrdlicka ZK, et al. Air bag-related ocular trauma. Am. J. Emerg. Med. 1997; 15:389-392.

${ }^{15}$ Larkin GL. Airbag-mediated corneal injury. Am. J. Emerg. Med. 1991; 9: 444-446. 
${ }^{16} \mathrm{O}$ Hullomun HS y cols. Primary enucleation as a consequence of airbag injury. J. Trauma, 44:190.

${ }^{17}$ Scott IU, John GR, Stark WJ. Airbag-associated ocular injury and periorbital fractures. Arch. Ophthalmol. 1993; 111: 25.

${ }^{18}$ Duma SM y cols. Airbag-induced eye injuries: a report of 25 cases. J. Trauma, 1996; 41: 114-119.

${ }^{19}$ Evans L. Restraint effectiveness, occupant ejection from cars and fatality reductions. Accid. Anal Prev. 1990; 22:167-75.

${ }^{20}$ Stein JD, Jaeger EA, Jeffers JB. Air bags and ocular injuries. TR. Am. Ophth. Soc. 1999: 59-86.

${ }^{21}$ Michalewska Z, Michalewski J, Nawrocka Z, Nawrocki J. Airbag hit as a cause of retinal detachment - case report. Klin. Oczna. 2007; 109:455-6.

${ }^{22}$ Whitacre MM, Pilchard WA. Air bag injury producing retinal dialysis and detachment. Arch. Ophthalmol. 1993; 111(10):132.

${ }^{23}$ Whitacre MM, Pilchard WA. Air bag injury producing retinal dialysis and detachment, Arch. Ophthalmol. 1993; 111:1320.

${ }^{24}$ Yang1Chang-Sue Yang1, Tzu-Fang Chou1, Jorn-Hon Liu, Wen-Ming Hsu1. Case report: air bag associated posterior segment ocular trauma. J. Chin. Med. Assoc. 2004; 67:425-431.

${ }^{25}$ Recommended procedures for evaluating occupant injury risk from deploying side airbags, the side airbag out-of-position injury technical working group (a joint project of alliance, aiam, aorc, and iihs). Adrian K. Lund, chairman, (first revision, 2003).

${ }^{26}$ Nightingale RW, Winkelstein BA. Injury mechanisms in the pediatric cervical spine during out-of-position airbag deployments. 42nd Annual Proceedings Association for the Advancement of Automotive Medicine, October 5-7, 1998 - Charlottesville, Virginia.

${ }^{27}$ Hallman JJ, Yoganandan N, Pintar FA. Torso side airbag out-of-position evaluation using stationary and dynamic occupants. Biomed. Sci. Instrum. 2008; 44: 123-128.

${ }^{28}$ Duma SM, Jernigan M, Virginia MS. The effects of airbags on orbital fracture patterns in frontal automobile crashes. Ophthalmic Plastic \& Reconstructive Surgery: March 2003; 9:107-111.

${ }^{29}$ Francis D, Kaufman R, Yueh B, Mock Ch, Nathens AB. Air bag-induced orbital blow-out fractures. The Laryngoscope, 2006; 116:1966-1972.

${ }^{30}$ Jin-Man Kim, Keun-Oh Kim, Young-Duk Kim, Gwang-Ju Choi. A case of air-bag associated severe ocular injury. Korean J. Ophthalmol., 2004; 18:84-88.

${ }^{31}$ Onwuzuruigbo, Chuks J. Fulda, Gerard J. Larned, David, Hailstone, Dalva. Case report: traumatic blindness after airbag deployment: bilateral lenticular dislocation, J. Trauma, 1996; 40: 314-316.

Resumo: Traumatismo ocular grave por airbag Descreve-se o caso de uma passageira do banco da frente de um automóvel que sofre um acidente (despiste com choque frontal direito contra barreira). A ocupante sofre fratura do pavimento da órbita direita e lesões oculares muito graves no olho direito, com rebenta- 
mento do globo ocular. Estas lesões, produzidas pelo airbag, foram facilitadas pela posição muito avançada do seu banco.

Palavras-chave: Acidente de tráfego; fratura do pavimento orbitário; lesão ocular grave; airbag; posição avançada do assento.

Abstract: Severe ocular injury by airbag

We report a case in which, the woman seating in the right front seat of a car that sustained a right-front crash against a fixed barrier, suffered severe facial injuries including a right orbital floor, and a blow-out injury to the right eye. These injuries were due to the airbag activation and probably were facilitated by an "out-of-position" - very near to the airbag - seating of the occupant.

Keywords: Traffic accident; orbital floor fracture; severe ocular injury; airbag; "out of position".

\section{Pedido de separatas:}

ANTONIO E. HERNANDO LORENZO

herloren@antonioehernando.e.telefonica.net 